\title{
Research on Situation Assessment Based on Fuzzy Algorithm in the Management of Unconventional Emergency
}

\author{
Xiaolin $\mathrm{LI}^{1, \mathrm{a}}{ }^{*}$,Chenggang $\mathrm{LI}^{2, \mathrm{~b}}$ \\ ${ }^{1}$ Business School, Sichuan Agriculture University Chengdu, China \\ ${ }^{2}$ Faculty of Finance, Guizhou University of Finance and Economics Guizhou, China \\ a57929606@qq.com, blichenggang603@126.com
}

Keywords: Fuzzy algorithm; Situation assessment; Unconventional emergency; Management

\begin{abstract}
The author applied fuzzy algorithm to a given situation assessment in unconventional emergency management process, then analyzed the mechanism of situation assessment in the emergency management. After that the author described the situation assessment process. Situation assessment model was established, and establish evaluation matrix of subordinate function.
\end{abstract}

\section{Introduction}

Today's world has entered the era with frequent incident. In 2008, after a snow disaster and 5.12 Wenchuan earthquake, 09 worldwide outbreak of succession H1N1 and Haiti earthquake, this year, natural disasters like Chile earthquake and Iceland volcanic eruption erupted. At the same time, social security, public health emergencies occur occasionally. So frequently and large-scale unconventional incident occurs, give our emergency management practice and research work a huge challenge and pressing demands. Especially in the abnormal events during the emergency decision-making and management, to the events of judgment, and select directly affects the further development of the situation. However, the judgment of situation plays a fundamental role in emergency decision and unconventional incident management process. High uncertainty of the incident is the fuzziness and randomness of the development of the events. Random refers to the development direction of the event with probability, such changes can be quantitative describe and process according to the theory of probability. And fuzzy logic can be natural fully express fuzzy information and knowledge, and easier to use by expert experience.

Based on this, the paper applied fuzzy algorithm to the emergency management situation assessment, and emphasizes the role of situation assessment mechanism and analyzed the application of the model, To make situation assessment more rapid and accurate, in order to provide reference. to emergency unconventional decision-making management[1] .

\section{Related Concepts}

Unconventional Emergency. At present, in the emergency management research, universal unconventional emergencies, Refers to the precursor is not full devastating and have the complexity of the obvious characteristic and the potential hazards of secondary derivatives, and difficult to handle with conventional management mode.

Situation Assessment. Situation assessment is mainly used in military field, is established on operational activities and events, time, place and force a piece of tissue of factor multiple views. It will connect the combat power distribution and the environment of the battle, identify already incidents and plan, to get the enemy forces structure, deployment, direction and route estimates, point out the enemy's behavior model, deduce the enemy's intentions, to make a reasonable explanation of the current battlefield situation, and to predict the change of the situation around time [2]. Huang Jun and some scholars think that scene is facing a kind of trend, the state refers to the current situation, is a result of the development in the past time, trend of the future is on the basis of the development In the current state to potential. Thus, in unconventional emergency management, Situation assessment is a process to establish a dynamic mechanism through in real-time, correctly 
processing information, and complete analysis and judge and predict the corresponding events, and output the results of analysis, judgement and forecasting immediately.

Fuzzy Transform and Evaluate Matrix. Fuzzy transform definition: Set $A \in F(X X Y), R \in$ $\mathrm{F}(\mathrm{X}), \mathrm{F}(\mathrm{H})=\mathrm{H}$ 。A, $\mathrm{f}$ is a fuzzy transform from the $\mathrm{X}$ to $\mathrm{Y}$. "。" Said generalized fuzzy synthetic operation [3].

Evaluate matrix: set of elements were evaluated for $X=(x 1, x 2, \ldots, x n)$, the level set by evaluation events for $\mathrm{Y}=(\mathrm{y} 1, \mathrm{y} 2, \ldots, \mathrm{yn})$. To assess this event by using fuzzy algorithm for single element, need to give the fuzzy mapping from $\mathrm{X}$ to $\mathrm{Y}$, like $\mathrm{f}: \mathrm{X} \rightarrow \mathrm{F}(\mathrm{y})$, $\mathrm{xi} \rightarrow \mathrm{f}(\mathrm{xi})=\mathrm{hi}=($ hi1,hi2,..,him $) \in \mathrm{F}(\mathrm{Y})$, it brings together all the elements of $\mathrm{n}$, thus can get a evaluation matrix $\mathrm{H}$ of $\mathrm{n}$ line and $\mathrm{m}$ column:

$$
\mathrm{H}\left(\begin{array}{c}
\mathrm{h}_{1} \\
\vdots \\
\mathrm{h}_{\mathrm{n}}
\end{array}\right)=\left(\begin{array}{cccc}
\mathrm{h}_{11} & \mathrm{~h}_{12} & \ldots & \mathrm{h}_{1 \mathrm{~m}} \\
\vdots & \vdots & & \vdots \\
\mathrm{h}_{\mathrm{n} 1} & \mathrm{~h}_{\mathrm{n} 2} & \ldots & \mathrm{h}_{\mathrm{nm}}
\end{array}\right)
$$

The formula expressed the fuzzy relation between level and the events evaluated [4]. The hi of the matrix reflects the membership first I for a set of elements evaluation, The first $\mathrm{j}$ column reflect each element of evaluated objects and take the first $j$ level of a set of evaluation, using statistical methods or empirical formula, determine the weight distribution of elements evaluated, operate by using fuzzy matrix composite fuzzy transform, is fuzzy evaluation for this event.

\section{Situation Assessment in the Emergency Management}

The Mechanism of Situation Assessment in Emergency Management. Regular event situation assessment is one-sided, interrupted, And irregular incident situation assessment circulate and repeat with the evolution of scene. Situation assessment and external mechanism mainly has four parts, to establish contact with four systems, including an output system (information system), used to provide information and data, two receiving system (decision-making system and evaluation system), used to receive the result of situation assessment, and a fixed system for modifying situation assessment results or decision scheme, as shown in Fig. 1.

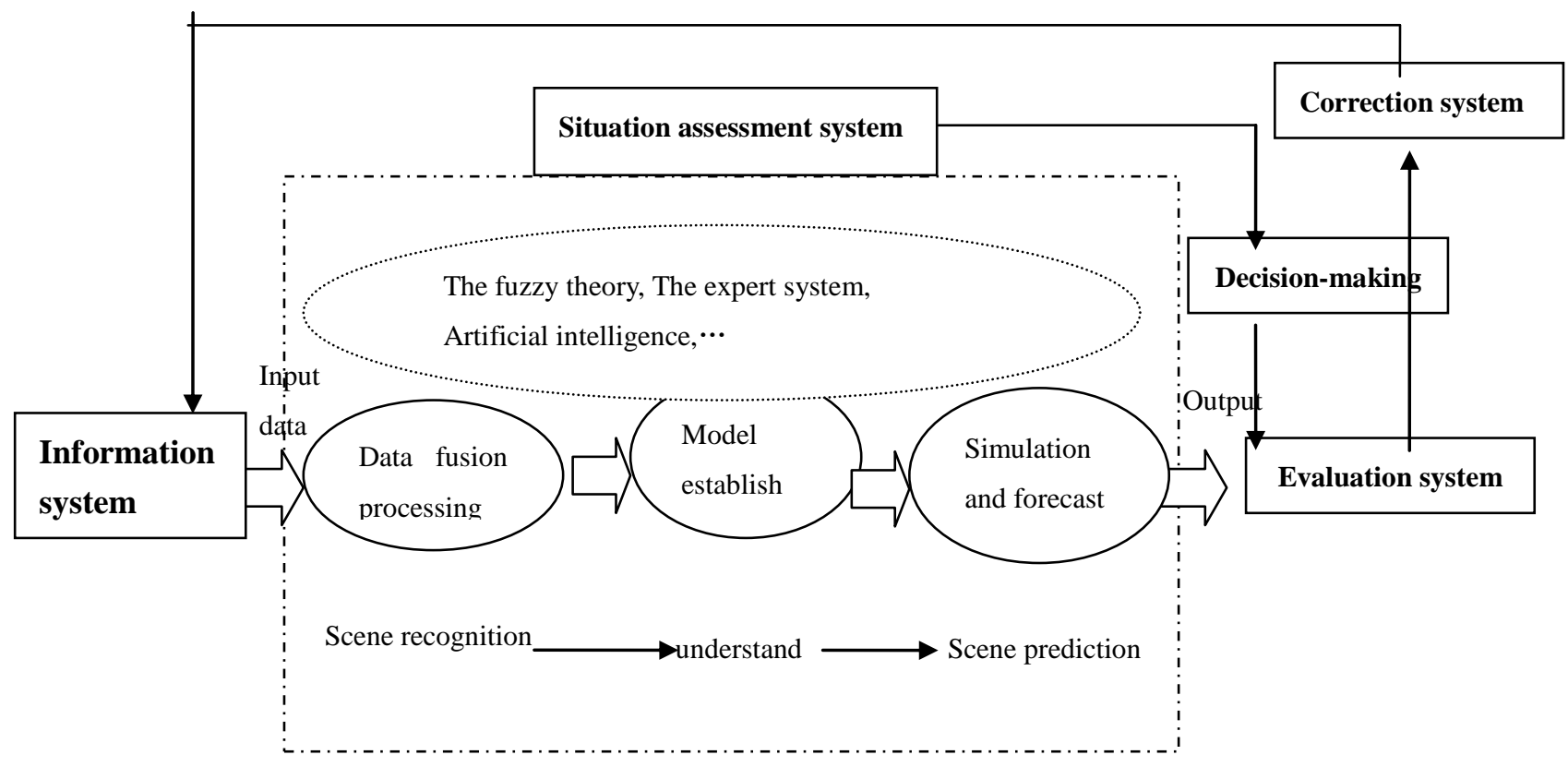

Figure 1. Situation assessment mechanism logic diagram

Situation Assessment Based on Fuzzy Algorithm. The outbreak and development of emergencies is a very complex and uncertain process, with unpredictable nature, one of the subtle elements may affect the development of the situation. Therefore, to evaluate the emergency 
situation, various situation elements and its global impact in evaluation objects involved should be considered by. And fuzzy comprehensive evaluation are effective methods of many factors [5], so we choose this kind of method to evaluate the emergency situation.

Set a set of elements from the scene of the incident for the situation $X=(x 1, x 2, \ldots, x n)$; The threat level set $\mathrm{Y}=(\mathrm{y} 1, \mathrm{y} 2, \ldots, \mathrm{ym})$; Evaluate matrix $\mathrm{H}=(\mathrm{h} 1, \mathrm{~h} 2, \ldots, \mathrm{hn}) \mathrm{T}$; the corresponding weights of Elements $d 1, d 2, \ldots, d n, d 1+d 2,+\ldots+d n=1$; Then said the $j$ with pj threat level yj on the credibility in X, Situation assessment model is concluded [6], as follows:

$$
\mathrm{p}_{\mathrm{j}}=\min \left\{1, \sum \operatorname{mind}_{\mathrm{i}=1} \mathrm{~m}_{\mathrm{j}} \mathrm{h}_{\mathrm{ij}}\right\}_{\circ}
$$

Set the threat level set $\mathrm{Y}=(\mathrm{y} 1, \mathrm{y} 2, \mathrm{y} 3, \mathrm{y} 4)$, The threat level diminish from $\mathrm{y} 1$ to $\mathrm{y} 4$, While to determine the evaluate matrix $\mathrm{H}$ by the membership function below [7].

$$
\begin{aligned}
& \mu_{\mathrm{y} 1}= \begin{cases}0, & 0<\mathrm{x} \leq 6.67 \\
(\mathrm{x}-6.67) / 3.33, & 6.67<\mathrm{x} \leq 10,\end{cases} \\
& \mu_{\mathrm{y} 2}=\left\{\begin{array}{cc}
0, & 0<\mathrm{x} \leq 3.33 \\
(\mathrm{x}-3.33) / 3.33, & 3.33<\mathrm{x} \leq 6.67 \\
(10-\mathrm{x}) / 3.33, & 6.67<\mathrm{x} \leq 10,
\end{array}\right. \\
& \mu_{\mathrm{y} 3}=\left\{\begin{array}{cc}
\mathrm{x} / 3.33, & 0<\mathrm{x} \leq 3.33 \\
(6.67-\mathrm{x}) / 3.33, & 3.33<\mathrm{x} \leq 6.67 \\
0, & 6.67<\mathrm{x} \leq 10
\end{array}\right. \\
& \mu \mathrm{y} 4=\left\{\begin{array}{cc}
(3.33-\mathrm{x}) / 3.33 & 0<\mathrm{x} \leq 3.33 \\
0 & 3.33<\mathrm{x} \leq 10
\end{array}\right.
\end{aligned}
$$

In the actual emergency management process, we according to the actual situation of the elements to specific situations and expert experience in $(0,10]$ interval determine the $\mathrm{x}$.

\section{Conclusions}

Using fuzzy algorithm, we can according to the real-time data and information and expert experience extract situation elements and set the threat level, measure conditional membership, also determine evaluation matrix of subordinate function parameter values, The calculates the threat level and evaluation of situation assessment on matrix elements in the situation of membership, determine the threat level.

\section{References}

[1] Han ZhiYong, Weng WenGuo, Zhang wei, Yangliexun, The major research plan "unconventional emergency management"of scientific research background, target and organizational management [J]. Chinese Science Fund.

[2] Hou Lin, Yang Fan, Zhang Zuo, Zhang yi. Study of situation assessment major traffic emergencies. Traffic Information and Safety, 2009, suppl 1 (27) 67-73.

[3] Jiang ZeJun, Fuzzy mathematics course, Defense industry press, 2004.

[4] LIN Xiao-qiang, CHANG Guo-cen, YANG Fan, YAN Hai-tao, Application of fuzzy algorithm in situation assessment Electronics Optics \& Control,2008,15(2)36-38. 
[5] LIN Wei-sheng,WANG Bao-shu, Situation assessment based on Bayesian networks [J]. Systems Engineering and Electronics, 2003,25(4):480-483.

[6] Wang Jinyi, Fuzzy equation and its application, Changsha, Hunan science and technology publishing house, 1991.

[7] Xing Chuandin, Yang Jaming, Ren Qinsheng, Artificial intelligence theory and application, Shanghai, Donghua university press, 2005. 